\title{
Ovarian Cancer Presenting as Cryptogenic Stroke from Patent Foramen Ovale
}

\author{
Bindu R. Potugari, MD; Priyanka Priyanka, MD; Sarah D. Komanapalli, MD; \\ and Richard J. Mercier, MD
}

\begin{abstract}
A woman, aged 52 years, experienced severe headache, confusion, nausea, dizziness, and diplopia for three days. Magnetic resonance imaging of the brain showed multiple acute and subacute infarcts suggestive of embolic events. Dermatological examination was notable for splinter hemorrhages and macular patches on the fingernails and feet, respectively. Further diagnostic imaging of the chest and abdomen revealed pulmonary emboli and an ovarian mass with omental deposits and splenic infarcts. Fine-needle aspiration cytology and surgery confirmed a diagnosis of high-grade serous adenocarcinoma of the ovary with clear cell features. Extensive evaluation for malignancy should be considered on a case-by-case basis for patients with thromboembolic disease and an initial negative diagnostic evaluation for stroke. Consideration of patent foramen ovale closure is reasonable in patients with malignancy who are at risk for recurrent strokes.
\end{abstract}

Keywords: Occult cancer; Ovarian mass; Ischemic stroke

A lthough the underlying etiologies are unknown, cryptogenic strokes account for $25 \%$ of ischemic strokes and tend to be thromboembolic in nature. ${ }^{1}$ Active cancer (presence of cancer before the diagnosis of stroke or within 12 months after index stroke) is associated with $2 \%$ to $12 \%$ of cryptogenic strokes. ${ }^{2,3}$ In rare cases, ischemic stroke can be the first manifestation of cancer. ${ }^{2,4}$ Malignancy increases the risk of venous thromboembolism (VTE) by seven-fold compared to the general population. ${ }^{5}$ Cancers associated with a higher incidence of stroke are lung, pancreatic, and colorectal cancers. ${ }^{3,6}$ VTE disease (pulmonary embolism [PE] and deep vein thrombosis [DVT]) are significantly associated with ovarian cancer $^{7}$ and adversely impact patient survival..$^{7-10}$ This case report outlines the discovery of occult ovarian cancer in a patient who exhibited acute cerebrovascular events with an incidental detection of patent foramen ovale (PFO).

\section{Case Presentation}

A Caucasian woman, aged 52 years, with a past medical history of hyperlipidemia and migraine headaches experienced severe frontal headache, photophobia, confusion, nausea, dizziness, and diplopia for 3 days. Her headaches were similar to migraine headaches but more severe in intensity. She was a nonsmoker and denied recreational drug use. Family history was positive for breast and rectal cancers in her mother and maternal grandfather, respectively. Her vital signs were stable except for an elevated blood pressure of 170 $\mathrm{mmHg} / 88 \mathrm{mmHg}$. A preliminary neurological examination revealed no significant focal neurologic deficits except for a delay in response to questions, and a computed tomography (CT) scan of the head showed no ischemic infarcts or hemorrhages. Normal heart sounds with no evidence of murmur were present during cardiovascular examination. However, the patient's initial hematology workup revealed elevated levels of leukocytes at $15.3 \times 10^{3}$ cells $/ \mu \mathrm{L}$. The patient was managed for a diagnosis of status migrainosus.

On day 3, the patient developed mild left upper extremity weakness with a motor strength of $4 / 5$ and cognitive dysfunction with trouble drawing a clock, difficulty performing simple math, and a Montreal Cognitive Scale 


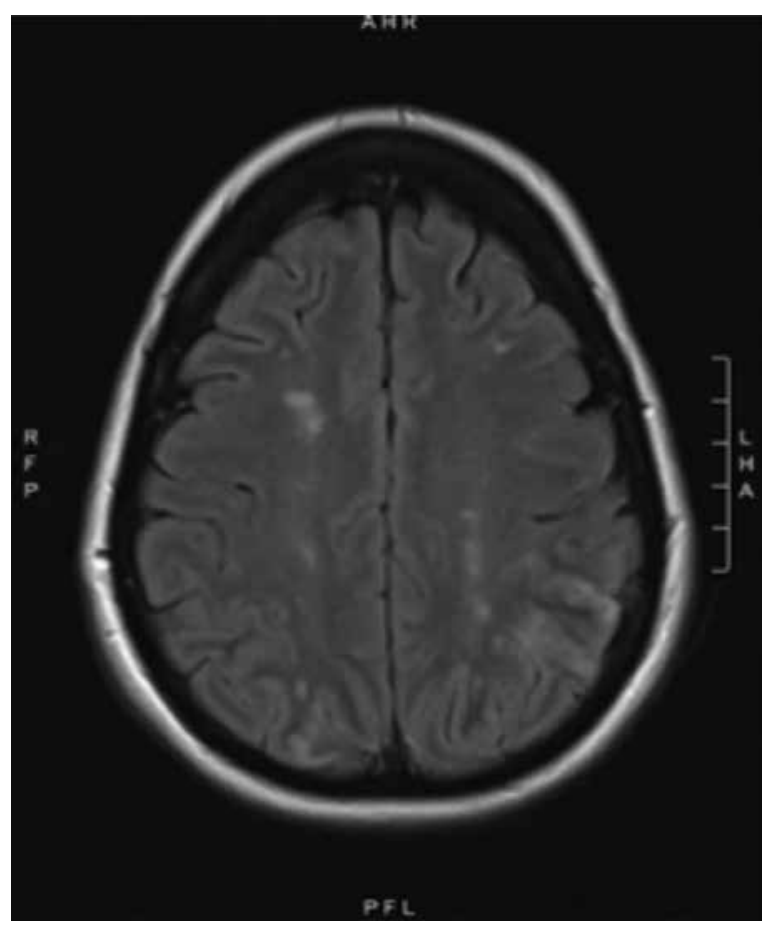

Figure 1. Brain magnetic resonance image revealing multiple infarcts.

score of $18 / 30$, indicative of cognitive impairment. With the development of new symptoms and persistent headaches, the patient was evaluated with magnetic resonance imaging of the brain, which highlighted acute and subacute infarcts in the watershed territory involving the frontal, parietal, and occipital lobes bilaterally with an extensive infarct in the left posterior parietal lobe (Figure 1).

A CT angiography of the patient's head and neck revealed no significant stenosis or vasculitis but incidentally found emboli in the right pulmonary artery. Her serial troponins were elevated at 1,596 ng/mL and 1,315 ng/mL (indicative of cardiac injury), although a transthoracic echocardiogram showed no PFO, vegetations, or right ventricular strain. A venous Doppler ultrasound of the patient's lower extremities detected acute thrombosis of the right lower extremity in the posterior tibial, deep muscular, and great saphenous veins. Anticoagulation was started with intravenous (IV) heparin.

On day 5, the patient developed subungual splinter hemorrhages in all fingernails and in a few toenails, purpuric macules, and retiform purpura in her distal fingers and toes (Figures 2 and 3). Pro-thrombotic and vasculitis workups were negative except for an elevated D-dimer of $5 \mathrm{mg} / \mathrm{L}$ with distant manifestations of thromboembolism in the skin and splenic infarcts. A transesophageal echocardiogram was performed to rule out endocarditis and showed a previously undetected PFO with right-to-left shunting and no vegetations (Figure 4). With her extensive thromboembolic disease, the patient was evaluated for occult malignancy with additional CT of the abdomen and pelvis, which highlighted a left ovarian mass, omental deposits, ascites, two splenic infarcts, and reactive left pleural effusion. An ultrasound of her pelvis confirmed the presence of a complex abnormal left ovary with cystic and heterogeneous solid components and diffuse internal vascularity. The contralateral ovary was unaffected based on imaging and histological investigations.

Ultrasound-guided fine-needle aspiration cytology of the omental deposits revealed an adenocarcinoma of mullerian origin, and serum testing of cancer markers revealed an elevated level of cancer antigen-125 (CA-125) at 294.1 U/mL. The patient was diagnosed with ovarian carcinoma FIGO stage III B, and her Khorana score was 1 prior to chemotherapy. With a family history of breast cancer in a first-degree relative, and as per Society of Gynecologic Oncology clinical practice and National Comprehensive Cancer Network guidelines, genetic testing for BRCA and p53 mutations was performed via OVaNext gynecological cancer testing panel, which was negative. With her significant clot burden and severe illness, the patient received six cycles of neoadjuvant chemotherapy with paclitaxel $\left(175 \mathrm{mg} / \mathrm{m}^{2}\right)$ and carboplatin $(6 \mathrm{mg} / \mathrm{AUC})$. She underwent surgical debulking with pelvic and aortic lymph node dissection and received three cycles of adjuvant chemotherapy with carboplatin (5 mg/AUC) and gemcitabine $\left(900 \mathrm{mg} / \mathrm{m}^{2}\right)$. Surgical pathology examination of the resected tissue revealed a left ovary with high-grade serous carcinoma with clear cell features and lymph nodal metastasis (Figure 5).

After multidisciplinary evaluation, the patient underwent percutaneous atrial septal repair with occluder device for PFO. She was anticoagulated with enoxaparin until 6 weeks after completion of adjuvant chemotherapy and continued aspirin for long-term secondary prophylaxis. She was disease-free 7 months after completion of adjuvant chemotherapy and showed significant improvement in neurologic function except mild confusion, occasional word-finding difficulties, and organizational issues. She resumed her work at 3 months after completion of adjuvant chemotherapy without any difficulty.

\section{Discussion}

Thromboembolism is one of the most common complications of malignancy that can occur before or after the diagnosis of

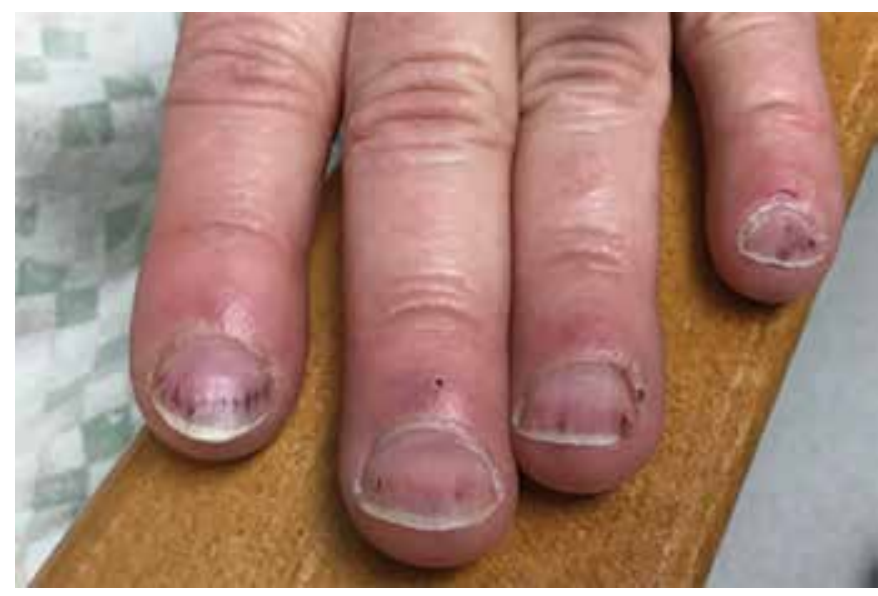

Figure 2. Splinter hemorrhages in fingernails 


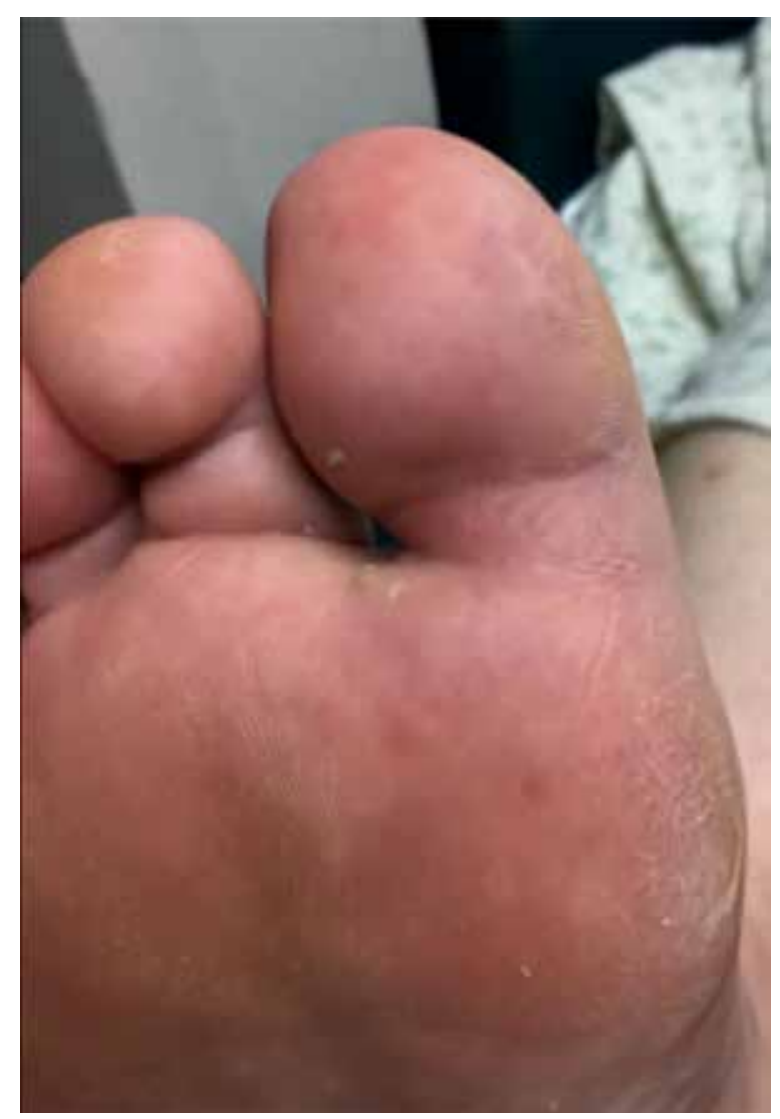

Figure 3. Macular patches on the foot.

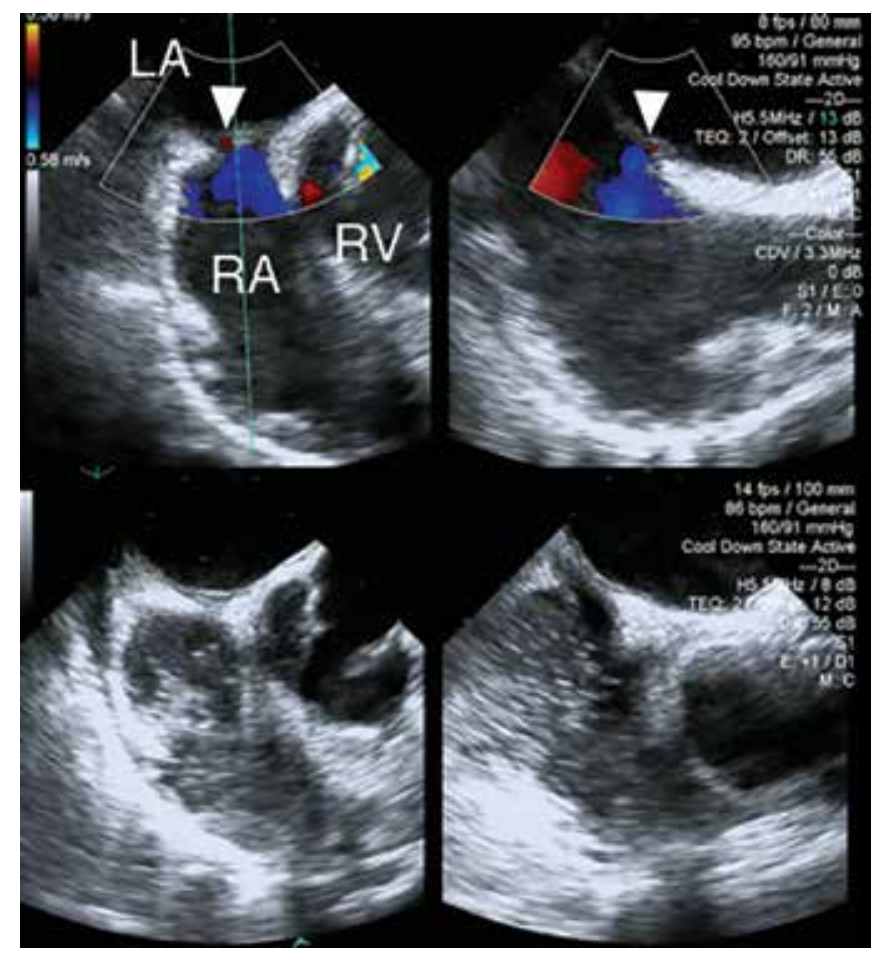

Figure 4. Transesophageal echocardiogram images showing positive agitated bubble study and right-to left shunting of blood flow with arrows pointing to the PFO. cancer. $^{7}$ A hypercoagulable state can occur in patients with cancer due to interactions between monocyte/macrophages with malignant cells causing the release of tumor factors such as tumor necrosis factor and interleukins- 1 and $-6 .^{11}$ This leads to endothelial damage and sloughing of endothelial cells, which acts as a thrombogenic surface to activate intrinsic and extrinsic coagulation factors that convert prothrombin to thrombin to generate thrombi. ${ }^{11}$ Other underlying clinical factors that can induce coagulation include advanced age, indwelling catheters, bedrest, hospitalization, and episodes of acute illness. ${ }^{5}$ According to Iodice and colleagues, ${ }^{12}$ ovarian, lung, pancreas, and liver cancers are associated with the highest risk of VTE. Initial studies in unprovoked VTE suggest that extensive occult cancer screening is necessary to detect more malignant conditions. ${ }^{13}$ With additional diagnostic workup, occult cancer is identified in one out of every 20 patients within a year of unprovoked VTE diagnosis, though there is insufficient evidence for screening all patients with unprovoked VTE for occult cancer to reduce cancer- and VTE-related morbidity and mortality. ${ }^{14,15}$ However, our patient's significant clot burden, cognitive decline, and presence of PFO warranted further diagnostic workup for occult cancer.

Venous thromboembolic events (PE/DVT) in gynecological malignancy range in incidence from $3 \%$ to $25 \% .^{7}$ VTE is either diagnosed incidentally during metastatic workup or may be symptomatic, with PE/DVT occurring immediately during the postoperative period and/or while undergoing active chemotherapy. VTE negatively impacts survival, which may indicate that thrombotic signaling promotes a more aggressive tumor behavior. ${ }^{9,16}$ Risk factors for VTE in ovarian cancer are advanced stage (III, IV), clear cell histology, chemotherapy, extensive cyto-reductive surgery, high-grade serous adenocarcinoma. ${ }^{10}$ Patients with VTE during primary treatment for ovarian cancer (clear cell) had a 3.9-fold increase in risk of recurrence and a 6.3-fold increase in risk of death, and VTE remained an independent prognostic factor for death, even after controlling the disease. ${ }^{16}$

The incidence of stroke as an initial manifestation of cancer varies in different studies and ranges from $0.4 \%$ to $10 \%{ }^{6,17}$ Strokes are most commonly associated with lung cancer but have occurred in other cancers as well. ${ }^{6,17}$ In patients with advanced adenocarcinoma, the risk of stroke recurrence is high. ${ }^{18}$ Factors that increase stroke risk in patients with cancer are Trousseau's syndrome, non-bacterial thrombotic endocarditis, disseminated intravascular coagulation, coagulopathies, changes in blood viscosity, leukocytosis, radiotherapy, and anticancer drugs. ${ }^{17,19}$ In a retrospective cohort analysis, Taccone and colleagues ${ }^{17}$ noted that patient outcomes associated with stroke and malignancy was poor with a median survival of 58 days. Based on results from two Norwegian registry-based studies, a clinical score was developed to predict the likelihood of active cancer in patients with ischemic stroke. ${ }^{2,4}$ This predictive score included elevated 

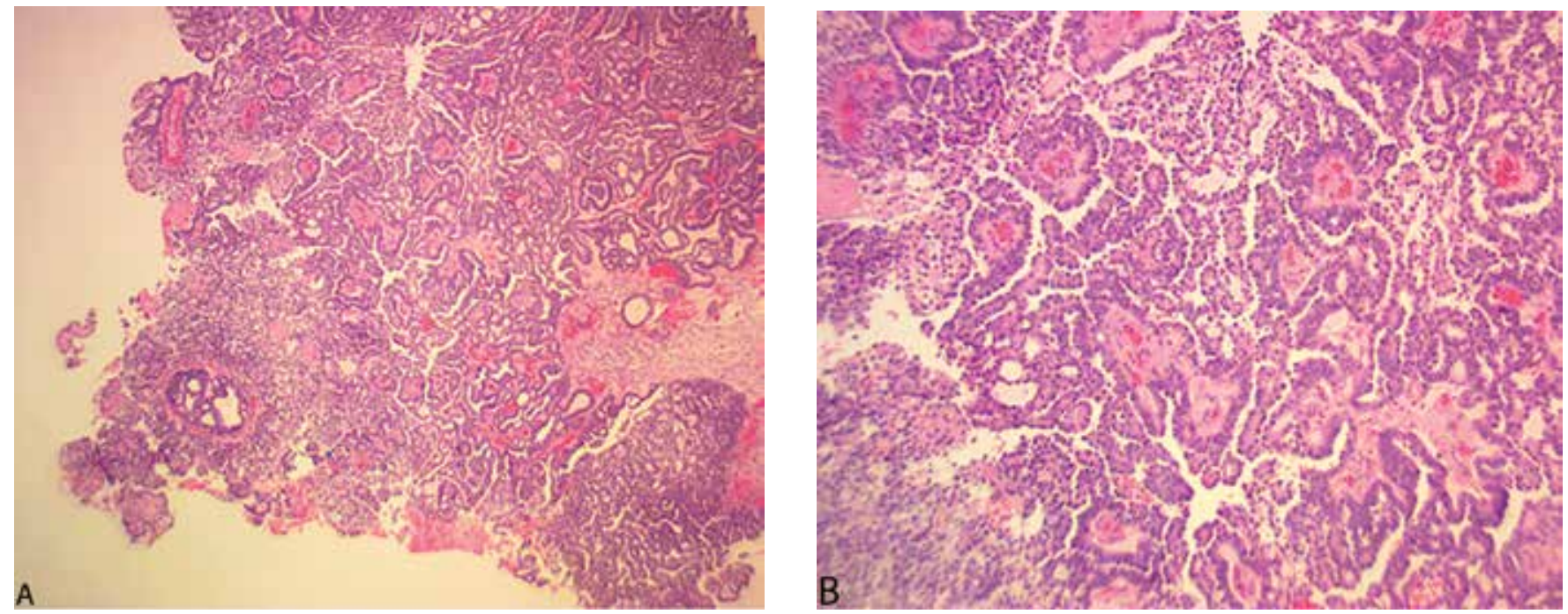

Figure 5. (Panel A \& B) Histopathology slides showing high grade serous ovarian carcinoma with clear cell features.

D-dimer levels ( $\geq 3 \mathrm{mg} / \mathrm{L})$, decreased hemoglobin levels ( $\leq 12$ $\mathrm{g} / \mathrm{dL}$ ), and a previous or current history of smoking. ${ }^{2}$

The importance of elevated D-dimer levels in identifying patients at increased risk for thromboembolism and cancer is supported by work by Gon et $\mathrm{al}^{6}$ and Kim et al. ${ }^{20}$ Both studies suggested elevated plasma D-dimer levels and lesions in multiple vascular regions might predict occult cancer in patients with cryptogenic stroke, ${ }^{6,20}$ while in a prospective study by Kim et al, ${ }^{21}$ high D-dimer level was associated with increased risk of recurrent ischemic stroke in patients with PFO. Our observation of elevated D-dimer levels and multiple infarcts in watershed areas of the brain in this case support these markers as a clue for evaluating patients with suspected malignancy-induced thromboembolism.

In addition to multiple cerebral infarcts, PE, and DVT, our patient developed peripheral manifestations of thromboembolism in the nailbeds, skin, and visceral organs during her hospital course, which indicated a possible occult malignancy requiring further diagnostic workup. During a transesophageal echocardiogram, a previously undetected PFO was discovered that was presumably the underlying cause of systemic embolization. Only two cases have been reported in the literature, to our knowledge, where PFO was both a cause for paradoxical embolism and an initial manifestation of ovarian cancer. ${ }^{22,23}$ In another case, a patient with ovarian cancer developed strokes during the postoperative period from an undetected PFO. ${ }^{24}$

Paradoxical embolism is the passage of a clot or other embolic particle from the venous circulation to the arterial circulation through a right-to-left shunt, such as an atrial or ventricular septal defect and pulmonary arteriovenous malformation..$^{25} \mathrm{~A}$ PFO is present in approximately one-quarter of the general patient population; however, in patients with cryptogenic stroke, this prevalence increases to one-half. ${ }^{26}$ Multiple randomized controlled trials suggest that closure of PFO in patients who have cryptogenic stroke secondary to PFO is beneficial in reducing the rate of recurrent strokes compared with antiplatelet or anticoagulant regimens. ${ }^{27,28}$ Our patient exhibited multiple paradoxical embolic sites including the skin, nailbeds, brain, and spleen, which raised suspicion for a possible PFO as the cause for embolism. Since the patient would remain hypercoagulable with her underlying malignancy, it was reasonable to consider PFO closure to prevent further thromboembolic events.

\section{Conclusion}

In summary, occult malignancy should be considered as a possible cause of cryptogenic stroke if the initial evaluation of stroke is negative. Diagnostic evaluation for occult cancer should be considered on a case-by-case basis in patients with unprovoked VTE and include measurement of serum D-dimer levels. Patients with involvement of multiple paradoxical embolic sites should raise suspicion for a possible PFO as the cause for embolism and be evaluated for PFO closure.

\section{Acknowledgement}

The authors would like to thank Emily Andreae, $\mathrm{PhD}$, for assistance with manuscript editing.

\section{References}

1. Hart RG, Diener HC, Coutts SB, et al; Cryptogenic Stroke/ESUS International Working Group. Embolic strokes of undetermined source: the case for a new clinical construct. Lancet Neurol. 2014;13(4):429-438.

2. Selvik HA, Bjerkreim AT, Thomassen L, Waje-Andreassen U, Naess H, Kvistad CE. When to screen ischemic stroke patients for cancer. Cerebrovasc Dis. 2018;45(1-2):42-47.

3. Gon Y, Okazaki S, Terasaki Y, et al. Characteristics of cryptogenic stroke in cancer patients. Ann Clin Transl Neurol. 2016;3(4):280-287.

4. Selvik HA, Thomassen L, Bjerkreim AT, Naess H. Cancerassociated stroke: The Bergen NORSTROKE Study. Cerebrovasc Dis Extra. 2015;5(3):107-113. 
5. Falanga A, Marchetti M, Vignoli A. Coagulation and cancer: biological and clinical aspects. J Thromb Haemost. 2013;11(2):223-233.

6. Gon Y, Sakaguchi M, Takasugi J, et al. Plasma D-dimer levels and ischaemic lesions in multiple vascular regions can predict occult cancer in patients with cryptogenic stroke. Eur J Neurol. 2017;24(3):503-508.

7. Cohen A, Lim CS, Davies AH. Venous thromboembolism in gynecological malignancy. Int J Gynecol Cancer. 2017;27(9):1970-1978.

8. Rodriguez AO, Wun T, Chew H, Zhou H, Harvey D, White RH. Venous thromboembolism in ovarian cancer. Gynecol Oncol. 2007;105(3):784-790.

9. Gunderson CC, Thomas ED, Slaughter KN, et al. The survival detriment of venous thromboembolism with epithelial ovarian cancer. Gynecol Oncol. 2014;134(1):73-77.

10. Saadeh FA, Norris L, O'Toole S, Gleeson N. Venous thromboembolism in ovarian cancer: incidence, risk factors and impact on survival. Eur J Obstet Gynecol Reprod Biol. 2013;170(1):214-218.

11. Bick RL. Cancer-Associated Thrombosis. N Engl J Med. 2003;349(2):109-111.

12. Iodice S, Gandini S, Löhr M, Lowenfels AB, Maisonneuve P. Venous thromboembolic events and organ-specific occult cancers: a review and meta-analysis. J Thromb Haemost. 2008;6(5):781-788.

13. Carrier M, Le Gal G, Wells PS, Fergusson D, Ramsay T, Rodger MA. Systematic review: the Trousseau syndrome revisited: should we screen extensively for cancer in patients with venous thromboembolism? Ann Intern Med. 2008;149(5):323-333.

14. van Es N, Le Gal G, Otten HM, et al. Screening for occult cancer in patients with unprovoked venous thromboembolism: A systematic review and meta-analysis of individual patient data. Ann Intern Med. 2017;167(6):410417

15. Robertson L, Yeoh SE, Stansby G, Agarwal R. Effect of testing for cancer on cancer- and venous thromboembolism (VTE)related mortality and morbidity in people with unprovoked VTE. Cochrane Database Syst Rev. 2017;8:CD010837.

16. Diaz ES, Walts AE, Karlan BY, Walsh CS. Venous thromboembolism during primary treatment of ovarian clear cell carcinoma is associated with decreased survival. Gynecol Oncol. 2013;131(3):541-545.

17. Taccone FS, Jeangette SM, Blecic SA. First-ever stroke as initial presentation of systemic cancer. J Stroke Cerebrovasc Dis. 2008;17(4):169-174.

18. Eschle D. Stroke and cancer: Are cryptogenic strokes a paraneoplastic syndrome? Praxis (Bern 1994). 2015;104:795802. [Article German]

19. Grisold W, Födinger M, Oberndorfer S. [Stroke in cancer patients. A paraneoplastic neurological syndrome?] Nervenarzt. 2010;81(4):418-422. [Article in German]

20. Kim SJ, Park JH, Lee MJ, Park YG, Ahn MJ, Bang OY. Clues to occult cancer in patients with ischemic stroke. PLoS One. 2012;7(9):e44959.

21. Kim YD, Song D, Nam HS, et al. D-dimer for prediction of long-term outcome in cryptogenic stroke patients with patent foramen ovale. Thromb Haemost. 2015;114(09):614-622.

22. Wada Y, Takahashi R, Yanagihara C, Nishimura Y. Paradoxical cerebral embolism as the initial symptom in a patient with ovarian cancer. J Stroke Cerebrovasc Dis. 2007;16(2):88-90.

23. Tadokoro Y, Sakaguchi M, Yagita Y, et al. Ischemic stroke in patients with solid gynecologic tract tumors and coagulopathy. Eur Neurol. 2013;70(5-6):304-307.

24. Mitsui T, Aoki Y, Nagata Y, Kojima Y, Tanaka K. Patent foramen ovale complicated by paradoxical embolism and brain infarct in a patient with advanced ovarian cancer. Gynecol Oncol. 2001;83(3):608-609.
25. Saver JL. Clinical Practice. Cryptogenic Stroke. N Engl J Med. 2016;374(21):2065-2074.

26. Saver JL. Cryptogenic stroke in patients with patent foramen ovale. Curr Atheroscler Rep. 2007;9(4):319-325.

27. Mas JL, Derumeaux G, Guillon B, et al; CLOSE Investigators. Patent foramen ovale closure or anticoagulation vs. antiplatelets after stroke. N Engl J Med. 2017;377(11):10111021.

28. Lattanzi S, Brigo F, Cagnetti C, Di Napoli M, Silvestrini M. Patent foramen ovale and cryptogenic stroke or transient ischemic attack: To close or not to close? A systematic review and meta-analysis. Cerebrovasc Dis. 2018;45(56):193-203.

\section{Author Affiliations}

Bindu R. Potugari, MD*; Priyanka Priyanka, MD ; Sarah D. Komanapalli, MDf; and Richard J. Mercier, MD\$

*Department of Internal Medicine, Marshfield Clinic, Marshfield, Wisconsin; Current affiliation: Oncology Fellow, St. Joseph Mercy Ann Arbor, Ypsilanti, Michigan †Department of Internal Medicine, Marshfield Medical Center, Marshfield, Wisconsin; Current affiliation: Department of Internal Medicine, University of West Virginia, Morgantown, West Virginia $¥$ Department of Hospital Medicine, Marshfield Medical Center, Marshfield, Wisconsin §Department of Oncology, Marshfield Medical Center, Marshfield, Wisconsin 